\title{
Aufruf zur Erfassung klinischer Studien in einem öffentlich zugänglichen Register
}

Die Unterzeichner dieses Aufrufes setzen sich dafür ein, dass in der Schweiz wie in anderen Ländern - jeder klinische Versuch in einem öffentlich zugänglichen Register erfasst wird. Eine solche Registrierung erscheint sowohl aus ethischen als auch aus wissenschaftlichen Gründen unverzichtbar; sie stellt eine umfassende Information über den Stand der wissenschaftlichen Erkenntnis sicher und bietet den Ärzten und Patienten leichter zugängliche Informationen zu neuen Behandlungsmöglichkeiten. Ein solches Vorhaben muss in enger internationaler Abstimmung umgesetzt werden, um dem globalen Charakter der klinischen Forschung gerecht zu werden.

Dieser Aufruf wird unterstützt durch

Prof. Peter M. Suter,

Präsident SAMW, Genf

Prof. Werner Stauffacher,

Vizepräsident SAMW, Basel

Prof. Peter Meier-Abt,

Vizepräsident SAMW, Zürich

Dr. Jacques de Haller,

Präsident FMH, Bern

Prof. Dieter Imboden,

Präsident des Nationalen Forschungsrates des Schweizerischen Nationalfonds, Zürich

Prof. André Perruchoud,

Präsident des «Collège des Doyens» der Medizinischen Fakultäten der Schweiz, Basel

sowie die Teilnehmer/innen des «Treffens der Repräsentanten von Institutionen im Bereich Klinische Versuche»,

26. Januar 2005, Bern
Alle an Menschen durchgeführten klinischen Studien unterliegen nationalen und internationalen Gesetzen, Standards und ethischen Prinzipien, die dazu dienen sollen, den Schutz der Studienteilnehmer und die Validität der Studiendaten sicherzustellen. Wesentlich ist dabei die Erwartung, dass der durch den persönlichen Einsatz von Patienten ermöglichte Erkenntnisgewinn uneingeschränkt für die Gesundheitsversorgung sowie für die Planung und Durchführung nachfolgender Studien zur Verfügung steht.

Dieser erwartete Erkenntnisgewinn kann durch eine verzögerte oder unterdrückte Publikation von abgebrochenen Studien oder Studien mit nicht erwünschten oder vermeintlich uninteressanten Ergebnissen (sogenannte negative Studien) eingeschränkt werden. Daraus kann eine Fehleinschätzung - meist eine zu optimistische - eines Arzneimittels oder eines nichtmedikamentösen Verfahrens resultieren. Zudem kann die Unkenntnis von geplanten oder durchgeführten Studien erhebliche negative Auswirkungen haben, indem zum Beispiel Risiken medizinischer Verfahren nicht hinreichend bekannt werden.

Klinische Studienregister können dazu beitragen, solche negativen Auswirkungen zu vermeiden und den Erkenntnisgewinn zu unterstützen, indem sie die Grundlage dafür bilden,

- Ärzte und Patienten sowie Institutionen der Gesundheitsversorgung über laufende Studien zu informieren und damit Studien durch schnellere Patientenaufnahme zu beschleunigen;

- die interessierte Öffentlichkeit transparent über klinische Studien zu informieren;

- redundante Studien zu vermeiden und somit die Durchführung anderer relevanter Studien zu ermöglichen;
- Kooperationen zwischen Studiendurchführenden zu fördern und höhere Aussagekraft durch grössere gemeinsame Studien zu ermöglichen;

- in Studien bereits aufgetretene Probleme und Erfahrungen öffentlich zu machen und bei der Planung neuer Studien zu berücksichtigen.

Aus all diesen Gründen wird die Erfassung sämtlicher klinischer Studien in einem öffentlich zugänglichen Register immer dringlicher gefordert. Mehrere nationale und globale Organisationen und Verleger wissenschaftlicher Zeitschriften haben sich dieser Forderung angeschlossen und registrieren eigene Studien bzw. machen eine Registrierung zur Bedingung für eine Publikation. In der Schweiz hat es bisher nur wenige isolierte Ansätze, jedoch keine umfassenden Schritte zur Studienregistrierung gegeben. Vor kurzem hat der Bundesrat darauf verzichtet, im Rahmen der Revision der Verordnung über klinische Versuche mit Heilmitteln entsprechende Vorgaben zu machen - dies, obwohl er von verschiedener Seite (u.a. Schweizerische Akademie der Medizinischen Wissenschaften, Schweizerische Gesellschaft für Chemische Industrie) darum gebeten worden war.

Die Unterzeichner dieses Aufrufes bitten die politischen Instanzen, die Erfassung klinischer Studien in einem öffentlich zugänglichen Register möglichst rasch für verbindlich zu erklären. Dabei wird zu klären sein, ob sich die Schaffung eines schweizerischen Registers aufdrängt (dies in enger Abstimmung und Kooperation mit internationalen Registern) oder ob die Anbindung an ein internationales, etabliertes Register vorzuziehen wäre.

In der Zwischenzeit rufen die Unterzeichner alle Studienleiter sowie alle öffentlichen und privaten Förderer auf, im Rahmen ihrer jeweiligen Pflichten und Möglichkeiten die Erfassung der in der Schweiz durchgeführten klinischen Studien in bestehenden Registern zu sichern. Sie ermuntern ferner alle anderen Beteiligten, wie z.B. Ethikkommissionen, Zulassungsbehörden und Träger forschender Einrichtungen, zur Durchsetzung der Registrierung in ihrem Tätigkeitsbereich beizutragen. 